DrAFt VERSION NOVEMBER 18, 2016

Preprint typeset using LATEX style AASTeX6 v. 1.0

\title{
THEORETICAL FOUNDATION OF 3D ALFVÉN RESONANCES: NORMAL MODES
}

\author{
Andrew N. Wright And Thomas Elsden \\ School of Mathematics and Statistics \\ University of St Andrews \\ Fife, KY16 9SS, UK
}

\begin{abstract}
We consider the resonant coupling of fast and Alfvén magnetohydrodynamic (MHD) waves in a 3D equilibrium. Numerical solutions to normal modes $(\propto \exp (-i \omega t))$ are presented along with a theoretical framework to interpret them. The solutions we find are fundamentally different to those in $1 \mathrm{D}$ and 2D. In 3D there exists an infinite number of possible resonant solutions within a "Resonant Zone," and we show how boundary conditions and locally 2D regions can favour particular solutions. A unique feature of the resonance in 3D is switching between different permissible solutions when the boundary of the Resonant Zone is encountered. The theoretical foundation we develop relies upon recognising that in 3D the orientation of the resonant surface will not align in a simple fashion with an equilibrium coordinate. We present a method for generating the Alfvén wave natural frequencies for an arbitrarily oriented Alfvén wave, which requires a careful treatment of scale factors describing the background magnetic field geometry.
\end{abstract}

Keywords: magnetohydrodynamics — planets and satellites: magnetic fields — Sun: magnetic fields - waves

\section{INTRODUCTION}

In this paper we address resonant coupling of fast and Alfvén waves in the low plasma $\beta$ limit in a background medium that depend upon all three spatial coordinates (3D). The theory has been well established in 1D by Southwood (1974) who showed that a resonant singularity existed on the field line where the Alfvén frequency $\left(\omega_{A}\right)$ is equal to the fast mode frequency (see also Chen \& Hasegawa (1974)) motivated by observations of the Earth's magnetosphere. There have also been simulations showing how 1D resonant solutions can grow with time (Allan et al. (1986), Mann et al. (1995)). Such wave coupling is a basic plasma process and also occurs in the solar context where 1D models have been used to explain the heating of coronal flux tubes (e.g., Goossens et al. (1995), Soler \& Terradas (2015), Arregui \& Ballester (2011), and references therein).

It is not obvious how the resonant behavior will generalise to $2 \mathrm{D}$, and indeed there were suggestions that the singularity in 1D was a quirk of the simplifications made in the 1D model (Hansen \& Goertz (1992)) and would not occur in 2D equilibria such as an axisymmetric magnetosphere or coronal arcade. The persistence of resonant wave coupling in 2D was established by Wright and Thompson (1994) and Russell and Wright (2010), and has also been considered in terms of quasi-modes by Andries et al. (2005). Naturally this raises the question of whether resonant coupling will occur in a 3D equilibrium. Some time-dependent simulations of waves in a 3D medium suggest that strong wave coupling will occur (Claudepierre et al. (2010), Degeling et al. (2010), Terradas et al. (2016)), but we are still lacking a theoretical foundation for understanding resonances in 3D.

To motivate our studies we consider the situation depicted in Figure 1, which uses transverse coordinates $\alpha$ and $\beta$ to act as labels for individual field lines. The plane in the figure can be envisaged as a cut perpendicular to the background magnetic field. For simplicity we could take the background magnetic field to be independent of $\beta$, but let the background density vary in 3D. The medium is further divided into three sections. In sections 1 and 3 the density is independent of $\beta$ so the medium here is completely 2D. In these regions we can exploit the results of Wright and Thompson (1994), and know we will excite resonant Alfvén waves at the red lines where the local Alfvén frequency matches the fast mode driving frequency $\left(\omega_{d}\right)$, and the Alfvén waves will have velocity and magnetic field perturbations polarised in the $\beta$ direction. In region 2 the density varies with $\beta$ so the medium is $3 \mathrm{D}$ here. How do the resonant fields in regions 1 and 3 connect through region 2, and is it appropriate to think of the solution in region 2 as "resonant"? 


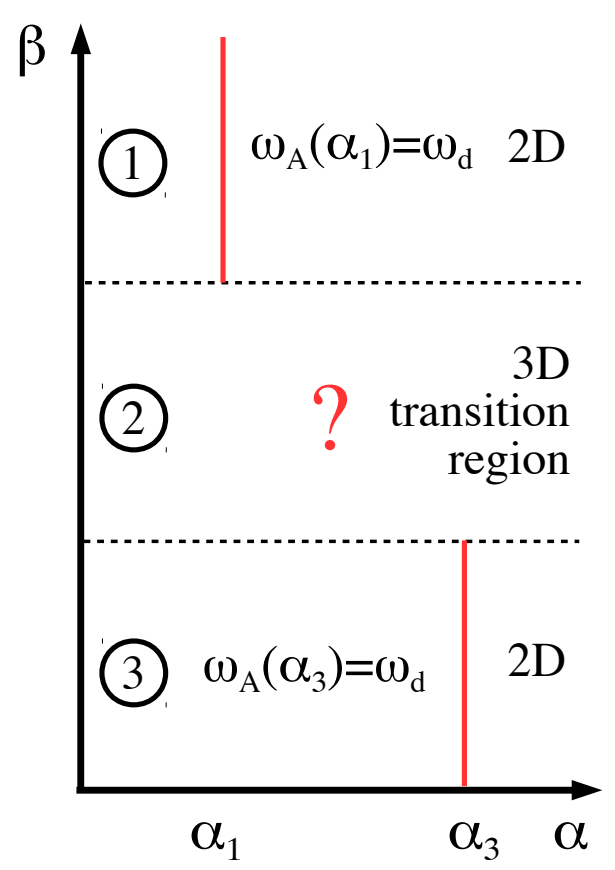

Figure 1. Variation of the Alfvén frequency $\left(\omega_{A}\right)$ with $\alpha$ and $\beta$ in a plane perpendicular to the background B. The red lines represent 2D resonance locations where the toroidal Alfvén frequency matches the driving frequency $\left(\omega_{d}\right)$.

To answer the above question we will provide numerical normal mode solutions (varying as $\exp (-i \omega t))$ to a system like that depicted in Figure 1. We make no attempt to model any particular physical system such as coronal structures or planetary magnetospheres accurately - although these are both situations which have motivated our study. Our emphasis is instead on designing numerical experiments that will give us the clearest understanding of the processes operating - something that would be compromised if a particular real system were modeled.

\section{ALFVÉN FREQUENCIES AND MAGNETIC GEOMETRY}

We begin by considering a similar equilibrium to that used in other studies in which the plasma pressure is neglected and a potential background magnetic field is used. Wright and Thompson (1994) used such an equilibrium and introduced a field aligned orthogonal coordinate system $(\alpha, \beta, \gamma)$ to investigate MHD wave coupling in which the unit vector $\mathbf{e}_{\gamma}$ is directed along the background magnetic field. Information about the magnetic field geometry is contained in scale factors $\left(h_{\alpha}, h_{\beta}\right.$ and $\left.h_{\gamma}\right)$ which are related to the real space element $d \mathbf{r}$ through

$$
d \mathbf{r}=\mathbf{e}_{\alpha} h_{\alpha} d \alpha+\mathbf{e}_{\beta} h_{\beta} d \beta+\mathbf{e}_{\gamma} h_{\gamma} d \gamma,
$$

Wright and Thompson (1994) show that Alfvén waves polarized in the $\alpha$ and $\beta$ directions satisfy the respective equations

$$
\begin{aligned}
& \frac{\partial}{\partial \gamma}\left(\frac{1}{h_{\gamma}} \frac{\partial U_{\alpha}}{\partial \gamma}\right)+\frac{1}{h_{\gamma}} \frac{\partial}{\partial \gamma}\left(\ln \left(\frac{h_{\alpha}}{h_{\beta}}\right)\right) \frac{\partial U_{\alpha}}{\partial \gamma}+\frac{\omega_{A}^{2}}{V^{2}} h_{\gamma} U_{\alpha}=0, \\
& \frac{\partial}{\partial \gamma}\left(\frac{1}{h_{\gamma}} \frac{\partial U_{\beta}}{\partial \gamma}\right)+\frac{1}{h_{\gamma}} \frac{\partial}{\partial \gamma}\left(\ln \left(\frac{h_{\beta}}{h_{\alpha}}\right)\right) \frac{\partial U_{\beta}}{\partial \gamma}+\frac{\omega_{A}^{2}}{V^{2}} h_{\gamma} U_{\beta}=0 .
\end{aligned}
$$

Here $U_{\alpha}=u_{\alpha} h_{\beta} B$ and $U_{\beta}=u_{\beta} h_{\alpha} B$, with $B$ being the background magnetic field strength, $V$ the background Alfvén speed, and $\mathbf{u}$ the plasma velocity.

For suitable boundary conditions (e.g., $\mathbf{u}=0$ at the ends of the field lines), equations (2) and (3) are Sturm-Liouville equations for the Alfvén frequency and Alfvén eigenfunction polarized in the $\alpha$ and $\beta$ directions. Note that, in the special case $h_{\alpha} \propto h_{\beta}$ on a given field line, the middle terms vanish and the two equations become identical yielding the same Alfvén frequencies and eigenfunctions. Geometrically such a magnetic field would have the property that the 
cross-sectional shape of a flux tube would be the same along the entire length of the tube (Wright (1990)). Except for very simple fields this is not the case, and in general we expect the Alfvén frequencies for the $\alpha$ and $\beta$ directions to be different.

A key concept used in our work is that on a given field line the Alfvén frequency will depend upon the polarisation of the Alfvén wave. Indeed, the more disparate the variation of $h_{\alpha}$ and $h_{\beta}$ is, the more the Alfvén eigenfrequencies for the $\alpha$ and $\beta$ direction are likely to differ. We shall choose a background magnetic field to enhance this difference. Indeed, we used a shooting code to calculate the two Alfvén frequencies of the fundamental modes for both a 2D dipole and a 3D dipole. For the 3D dipole the $\alpha$ and $\beta$ Alfvén frequencies for a field line with footpoints at $77^{\circ}$ latitude differed by around $40 \%$. For the $2 \mathrm{D}$ dipole the frequencies differ by a factor of 3 (Elsden (2016)). This is consistent with the fact that the ratio of $h_{\alpha}$ to $h_{\beta}$ varies more for the $2 \mathrm{D}$ dipole than the $3 \mathrm{D}$ dipole. For this reason we shall adopt the 2D dipole in our numerical solutions.

A 2D potential dipole lying along the $z$ axis in cylindrical $(R, \phi, z)$ coordinates may be described in terms of the scalar and vector potentials, $\mathbf{B}=\boldsymbol{\nabla} \psi=\boldsymbol{\nabla}_{\times}\left(\mathbf{e}_{z} A\right)$

$$
\psi=\frac{B_{o} R_{o}^{2}}{R} \sin \phi, \quad A=\frac{A_{o} R_{o}}{R} \cos \phi, \quad A_{o}=B_{o} R_{o}
$$

and $A$ corresponds to the $z$ component of the vector potential. At $(R, \phi)=\left(R_{o}, 0\right)$ the background field strength is $B_{o}$ and $A=A_{o}$.

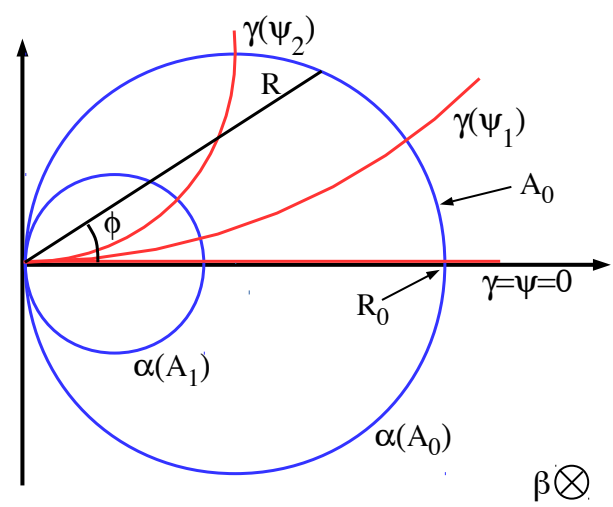

Figure 2. Lines of constant $A$ (blue) correspond to background magnetic field lines, whilst lines of constant scalar potential $\psi$ (red) provide a field aligned coordinate.

Figure 2 shows the magnetic field lines in blue, which coincide with lines of constant $A$ and provides a natural coordinate to label field lines. The red lines indicate lines of constant $\psi$ which act as a natural field aligned coordinate. The remaining coordinate could be chosen as $z$. The problem with using $(A, z, \psi)$ as coordinates is that a uniformly spaced grid in $A$ and $\psi$ will be highly nonuniform in real space leading to an inefficient numerical solution. Instead we choose alternative coordinates $\alpha(A), \beta=z$ and $\gamma(\psi)$ so that the coordinate surfaces remain like those in Figure 2, but a uniform grid in $\alpha, \beta$ and $\gamma$ produces a more even grid in real space. Specifically we take

$$
\alpha=\frac{R}{\cos \phi}, \quad \beta=z, \quad \gamma=R_{g} \tan ^{-1}\left(\frac{R_{g}}{R} \sin \phi\right) .
$$

Here $R=R_{g}$ is a reference point on the $\psi=0$ surface and is chosen so that the field line passing through it is roughly in the centre of our simulation domain. For this field line our choice of $\gamma(\psi)$ has the property that $\gamma$ is equal to the distance along that field line from the point where $\psi=0$. Hence a uniform grid in $\gamma$ will produce a reasonably uniform grid along the field line in real space. We also note that in the $\gamma=\psi=0$ plane the two transverse coordinates $\alpha$ and $\beta$ coincide with $R$ and $z$, respectively, so correspond to real lengths. This facilitates the interpretation of our results, in addition to providing a uniform real space grid in this plane for uniform $\alpha$ and $\beta$ grids. The numerical solution is calculated completely in $(\alpha, \beta, \gamma)$ space for which the corresponding scale factors are (see Elsden (2016))

$$
h_{\alpha}=\frac{1}{1+\left(\alpha / R_{g}\right)^{2} \tan ^{2}\left(\gamma / R_{g}\right)}, \quad h_{\beta}=1, \quad h_{\gamma}=\frac{1}{\left(R_{g} / \alpha\right)^{2} \cos ^{2}\left(\gamma / R_{g}\right)+\sin ^{2}\left(\gamma / R_{g}\right)}
$$


Note that the origin represents a singular point in our coordinate system, however this need not cause concern: the type of potential fields we consider would be generated by point source multipoles located at the origin and will not be contained in the domain where we solve the governing MHD equations. For example, in the case of a simple planetary magnetosphere the multipoles would lie inside the planet. Similarly, for a coronal arcade the multipoles would lie beneath the photosphere.

\section{3D ALFVÉN RESONANCES}

\subsection{Governing linear equations}

In this subsection we present the governing linear equations to our model, which are solved in the field aligned coordinate system described above. Neglecting plasma pressure the governing equations for linear perturbations are

$$
\begin{gathered}
\frac{\partial U_{\alpha}}{\partial t}=V^{2} \frac{h_{\beta}}{h_{\alpha} h_{\gamma}}\left[\frac{\partial B_{\alpha}}{\partial \gamma}-\frac{\partial B_{\gamma}}{\partial \alpha}\right]-\nu U_{\alpha} \\
\frac{\partial U_{\beta}}{\partial t}=V^{2} \frac{h_{\alpha}}{h_{\beta} h_{\gamma}}\left[\frac{\partial B_{\beta}}{\partial \gamma}-\frac{\partial B_{\gamma}}{\partial \beta}\right]-\nu U_{\beta} \\
\frac{\partial B_{\alpha}}{\partial t}=\frac{h_{\alpha}}{h_{\beta} h_{\gamma}} \frac{\partial U_{\alpha}}{\partial \gamma} \\
\frac{\partial B_{\beta}}{\partial t}=\frac{h_{\beta}}{h_{\alpha} h_{\gamma}} \frac{\partial U_{\beta}}{\partial \gamma} \\
\frac{\partial B_{\gamma}}{\partial t}=-\frac{h_{\gamma}}{h_{\alpha} h_{\beta}}\left[\frac{\partial U_{\alpha}}{\partial \alpha}+\frac{\partial U_{\beta}}{\partial \beta}\right]
\end{gathered}
$$

For numerical convenience the equations are cast in terms of the fields $U_{\alpha}, U_{\beta}, B_{\alpha}, B_{\beta}$ and $B_{\gamma}$, where $U_{\alpha}=u_{\alpha} h_{\beta} B$, $U_{\beta}=u_{\beta} h_{\alpha} B, B_{\alpha}=b_{\alpha} h_{\alpha}, B_{\beta}=b_{\beta} h_{\beta}$, and $B_{\gamma}=b_{\gamma} h_{\gamma}$. Here $\mathbf{u}$ is the plasma velocity, $\mathbf{b}$ the magnetic field perturbation, $V$ is the Alfvén speed $\left(B / \sqrt{\mu_{o} \rho}\right), B$ is the equilibrium field strength, and $\rho$ the equilibrium density. In these equations lengths have been normalised by $R_{o}$, magnetic fields by $B_{o}=B\left(R=R_{o}, \phi=0\right)$, density by $\rho_{o}=\rho\left(R=R_{o}, \phi=0\right)$, velocity by $V_{o}=V\left(R=R_{o}, \phi=0\right)$, and time by $R_{o} / V_{o}$. We have included a small amount of dissipation in the form of a linear drag term $(\nu)$ to prevent any singularity in our solution. We shall consider steady oscillatory solutions to the above equations that vary as $\exp (-i \omega t)$. Time dependent solutions are presented in a companion paper (Elsden \& Wright (2016)).

\subsection{Numerical Details}

We discretise the above equations (after replacing $\partial / \partial t$ with $-i \omega$ ) on a staggered grid with constant spacing $\Delta \alpha, \Delta \beta$ and $\Delta \gamma$ : if a unit elemental volume has $B_{\gamma}$ defined at $(0,0,0)$, then $U_{\alpha}$ is defined at $(\Delta \alpha / 2,0,0), U_{\beta}$ at $(0, \Delta \beta / 2,0)$, $B_{\alpha}$ at $(\Delta \alpha / 2,0, \Delta \gamma / 2)$, and $B_{\beta}$ at $(0, \Delta \beta / 2, \Delta \gamma / 2)$. The equations are solved over the domain $\alpha_{\min }<\alpha<\alpha_{\max }$, $\beta_{\min }<\beta<\beta_{\max }$ and $\gamma_{\min }<\gamma<\gamma_{\max }$.

The boundary at $\alpha_{\max }$ is used to drive the system by specifying $b_{\gamma}$ on it, which represents forcing the system with a magnetic pressure perturbation. At the other boundaries we impose nodes or antinodes of the fields which provides perfectly reflecting boundaries. Specifically: $\alpha=\alpha_{\text {min }}$ has nodes (antinodes) of $b_{\alpha}, u_{\alpha}\left(u_{\beta}, b_{\beta}, b_{\gamma}\right) ; \beta=\beta_{\text {min }}$ and $\beta_{\text {max }}$ have nodes (antinodes) of $b_{\beta}, u_{\beta}\left(u_{\alpha}, b_{\alpha}, b_{\gamma}\right) ; \gamma=0$ has nodes (antinodes) of $b_{\alpha}, b_{\beta}\left(u_{\alpha}, u_{\beta}, b_{\gamma}\right) ; \gamma=\gamma_{\text {max }}$ has nodes (antinodes) of $u_{\alpha}, u_{\beta}, b_{\gamma}\left(b_{\alpha}, b_{\beta}\right)$.

For the solutions presented in this section the domain is taken to be $0.5<\alpha<1.0,-0.2<\beta<1.0$ and $0<\gamma<0.525$ with $R_{g}=0.75$. The boundary condition at $\gamma=0$ can also be viewed as a symmetry condition, allowing our solutions to be interpreted as the fundamental (and odd harmonics) of the extended domain $-\gamma_{\max }<\gamma<\gamma_{\max }$. We let the Alfvén speed $(V)$ be constant along any given field line, but allow it to have a linear variation with $\beta$. The result is an equilibrium that varies with $(\alpha, \beta, \gamma)$ and allows us to study $3 \mathrm{D}$ resonances. We also introduce buffer zones for $\beta<-0.05$ and $\beta>0.85$ where the dissipation coefficient $(\nu)$ ramps up smoothly to 1.0 , meaning the solution is quite insensitive to the boundary conditions at $\beta_{\min }$ and $\beta_{\max }$ and allows us to drive the $\alpha_{\max }$ boundary with a sinusoidal magnetic pressure perturbation that propagates in the $+\beta$ direction. The wavenumber in $\beta$ is taken to be 10.0 and the angular frequency was 2.1647. For the subdomain of interest $0<\beta<0.85$ (which excludes the buffer zones) this essentially represents part of an open-ended waveguide with a boundary driver running down the waveguide. The 
variation of $b_{\gamma}$ on the driven boundary with $\gamma$ is chosen to represent forcing centred on $\gamma=0$ and being zero for $\gamma>\gamma_{\max } / 2$. Outside of the buffer zones the drag coefficient $\nu=0.03$. The finite difference equations are solved on a grid $(150 \times 250 \times 25)$ using a direct solver. It is also possible to remove any resonant singularity by setting $\nu=0$ outside of the buffer zones and introducing a small positive imaginary part to $\omega$. If $\omega_{i}$ is chosen appropriately these results (not shown here) gave the same features as for results with $\nu \neq 0$ and $\omega$ real.

To check the accuracy of the solution we estimated how well the solenoidal constraint on the perturbation magnetic field was satisfied. The surface integral of $b_{n}$ (the normal magnetic field component) was calculated over the simulation domain and compared to the surface integral of $\left|b_{n}\right|$, indicating the field was divergence free to typically 1 part in $10^{5}$ or better. We also estimated how well energy continuity was satisfied based upon balancing the surface integral of the time-averaged Poynting vector and the volume integral of the time-averaged dissipation due to the drag term. For the results in this paper energy continuity was typically satisfied to between $1 \%$ and $4 \%$.

To interpret the solutions we calculate the time-averaged total energy density $(W)$ in real space. In terms of the fields we solve for this has the form

$$
W=\frac{1}{4}\left[\frac{U_{\alpha} U_{\alpha}^{*}}{V^{2} h_{\beta}^{2}}+\frac{U_{\beta} U_{\beta}^{*}}{V^{2} h_{\alpha}^{2}}+\frac{B_{\alpha} B_{\alpha}^{*}}{h_{\alpha}^{2}}+\frac{B_{\beta} B_{\beta}^{*}}{h_{\beta}^{2}}+\frac{B_{\gamma} B_{\gamma}^{*}}{h_{\gamma}^{2}}\right]
$$

A signature of an Alfvén resonance is the accumulation of energy at particular "resonant" locations. Figure 3(a) shows a shaded surface plot of $W(\alpha, \beta, \gamma=0)$, and the line along which $W$ is concentrated is evident. A key property of an Alfvén resonance is that it should extend along the field lines, since a resonant field line will be resonant along its entire length. To check this we have plotted the locus of the ridge in Figure 3(a) as the red line in Figure 3(b). We then produced a similar plot to that in (a) for the plane $\gamma=\gamma_{\max }$, calculated the path of the ridge, and have displayed it as the dashed blue line in Figure 3(b). The two ridges lie exactly on top of one another. Indeed the same result was found for intermediate $\gamma$ planes (not shown), clearly indicating that our solution does indeed have a favoured sheet of field lines on which the energy is accumulated. This highlights the efficacy of working in $(\alpha, \beta, \gamma)$ space. Figure 3(c) shows the surface containing the field lines where energy accumulates together with a skeleton frame of the simulation domain in physical space (rather than $(\alpha, \beta, \gamma)$ space). We also note that the amplitude of the energy ridge tends to decrease with distance from the driven $(\alpha=1)$ boundary - presumably due the the evanescence of the fast mode.

Solutions for resonant Alfvén waves in $1 \mathrm{D}$ and $2 \mathrm{D}$ show that $\mathbf{b}_{\perp}$ and $\mathbf{u}_{\perp}$ lie in the resonant surface in real space. We can check if this is true for our solution by focusing on the region indicated by the red square in Figure $3(\mathrm{a})$. The alignment needs to be examined in real space, so we integrate (1) to find the associated distances along the $\alpha$ and $\beta$ directions to be

$$
r_{\alpha}=\frac{R_{g}}{\tan \left(\gamma / R_{g}\right)} \tan ^{-1}\left(\frac{\alpha}{R_{g}} \tan \left(\gamma / R_{g}\right)\right)+\text { const., } \quad r_{\beta}=\beta+\text { const. }
$$

and choose the constants to measure distance from the $\alpha=\alpha_{\min }$ surface and the $\beta=0$ surface.

Figure 4 shows color plots of $W$ to identify the location where energy is accumulated: panel (a) is plotted in the $\gamma=0$ plane and the arrows (representing $\mathbf{u}_{\perp}$ ) confirm that $\mathbf{u}_{\perp}$ here is aligned with the resonance. In the $\gamma=\gamma_{\max }$ plane (shown in (b)) we see that $\mathbf{b}_{\perp}$ is also aligned with the resonant surface. Other simulations (not shown) confirmed that the amplitude of the resonant fields and the width of the resonant layer scaled as $1 / \nu$ and $\nu$, respectively. All these features are characteristic of an Alfvén resonance.

\section{3D RESONANCE CONJECTURE}

The results shown in the previous section strongly suggest there is an Alfvén resonance present. However, the 3D resonant surface is not simply aligned with one of the coordinate directions, and there is no understanding of how to predict the location of the sheet on which energy accumulates in 3D. We anticipate that there will be some matching of the Alfvén frequency with the driving frequency, but it will not be simply the poloidal or toroidal frequency found from equations (2) and (3). Figures 3 and 4 clearly indicate the Alfvén resonance has an intermediate (and varying) polarisation.

To calculate the Alfvén frequency for an Alfvén wave polarised at an arbitrary angle we adopt the ideas used in Singer et al. (1981) who used orthogonal field aligned coordinates whose transverse coordinates are aligned with the direction of the Alfvén wave polarisation. Indeed such a coordinate system has been exploited previously by Russell and Wright (2010). The problem is then reduced to that of calculating the appropriate scale factors for this new coordinate system, and is addressed in the following subsection. 

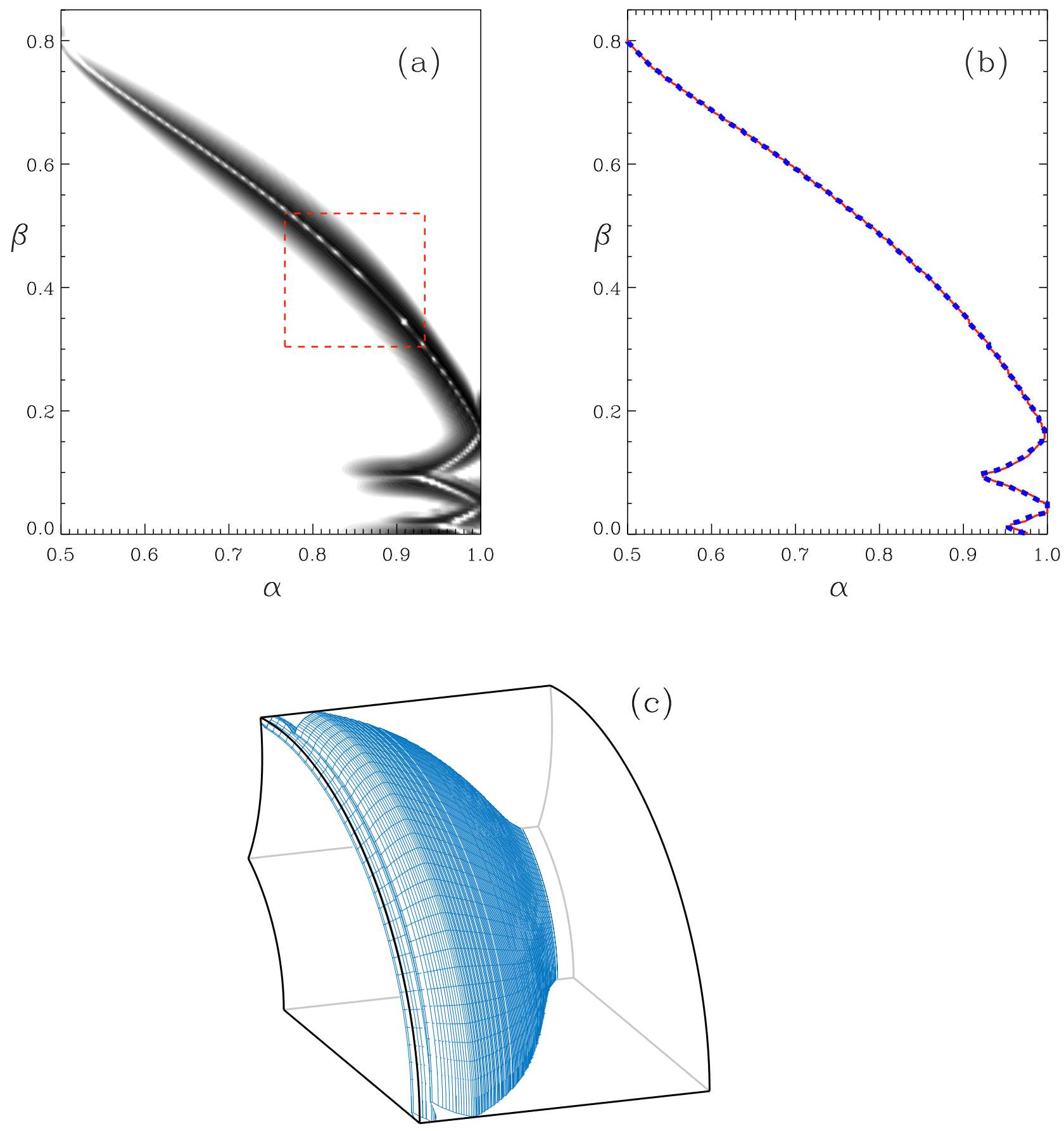

Figure 3. (a) Variation time-averaged energy density with $\alpha$ and $\beta$ in the $\gamma=0$ plane. (b) Peak energy density path (red line, $\gamma=0$; blue dashed line $\gamma=\gamma_{\max }$ ). (c) A skeleton frame depicting the simulation domain in physical space. Also shown is the surface formed by the sheet of field lines on which energy accumulates.

\subsection{Scale Factors}

Figure 5 shows the intersection of two field lines (denoted by the red dots labelled $p$ and $q$ ) with a $\gamma=$ constant surface in (a) coordinate space $(\alpha, \beta)$, and (b) real space $\left(r_{\alpha}, r_{\beta}\right)$. If $p$ and $q$ both lie on the resonant ridge, we can define two new local transverse coordinates $\left(\alpha^{\prime}, \beta^{\prime}\right)$ which are orthogonal and have the points $p$ and $q$ lying on the $\beta^{\prime}$ axis. The angle between the $\beta$ and $\beta^{\prime}$ axes is $\theta$, as shown in Figure 5(a). Evidently 

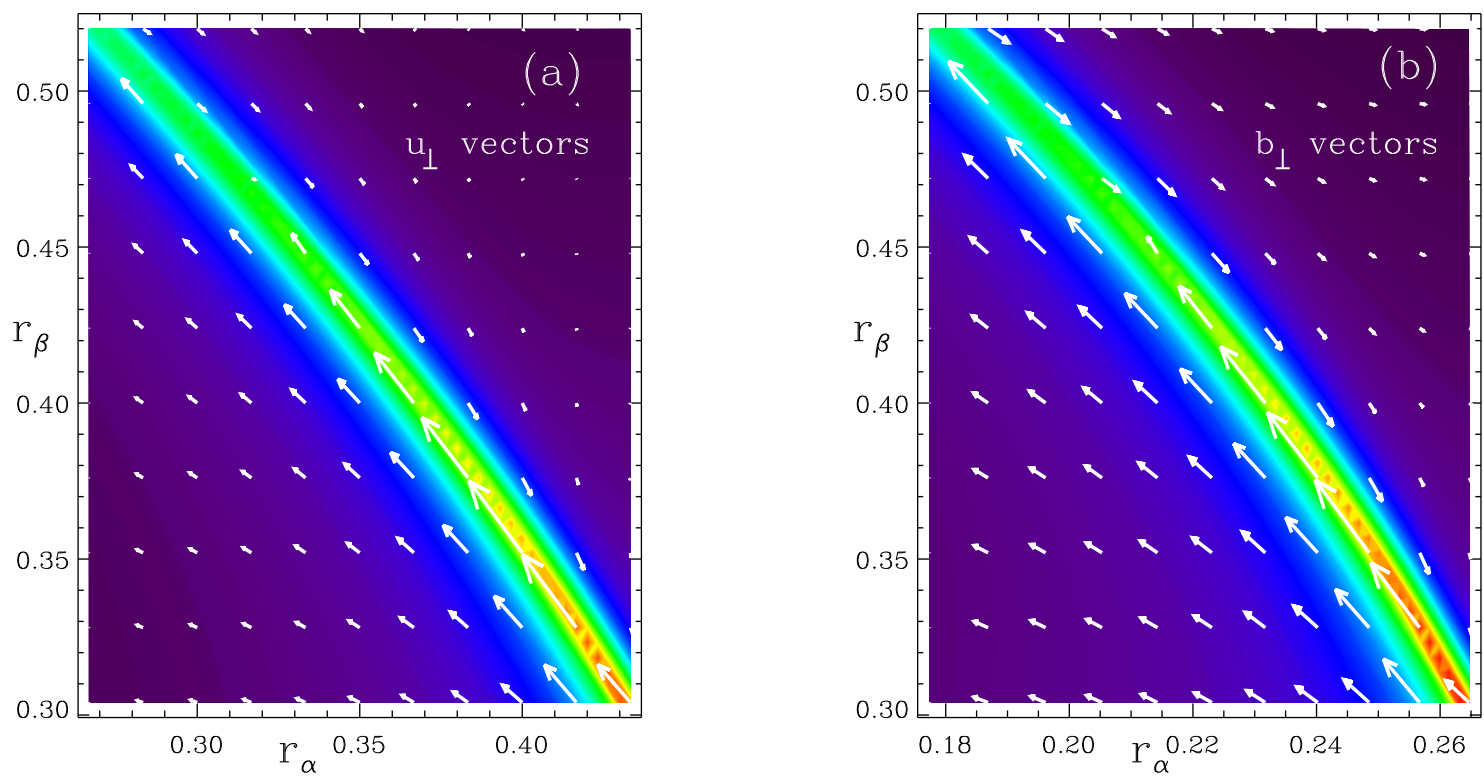

Figure 4. A close up of the region in the dashed rectangle of Figure 3(a). Colors indicate energy density and arrows denote $\mathbf{u}_{\perp}$ in the $\gamma=0$ plane (a) or $\mathbf{b}_{\perp}$ in the $\gamma=\gamma_{\max }$ plane (b). The axes correspond to distances along the $\alpha$ axis and the $\beta$ axis.

$$
\frac{d \alpha}{d \beta}=\tan \theta
$$

In the physical space $\left(r_{\alpha}, r_{\beta}\right)$ plane, the angle the line $p q$ makes to the $r_{\beta}$ axis is (Figure $\left.5(\mathrm{~b})\right) \theta_{\mathbf{r}}$

$$
\tan \theta_{\mathbf{r}}=\frac{h_{\alpha}}{h_{\beta}} \frac{d \alpha}{d \beta} \quad \Rightarrow \quad \tan \theta_{\mathbf{r}}=\frac{h_{\alpha}}{h_{\beta}} \tan \theta
$$

Note that $\theta$ is independent of $\gamma$, but $\theta_{\mathbf{r}}$ is not, meaning the resonant surface twists and rotates in real space as we move along a field line, whereas it maintains a fixed orientation in $(\alpha, \beta)$ space - as shown in Figure $3(\mathrm{~b})$.
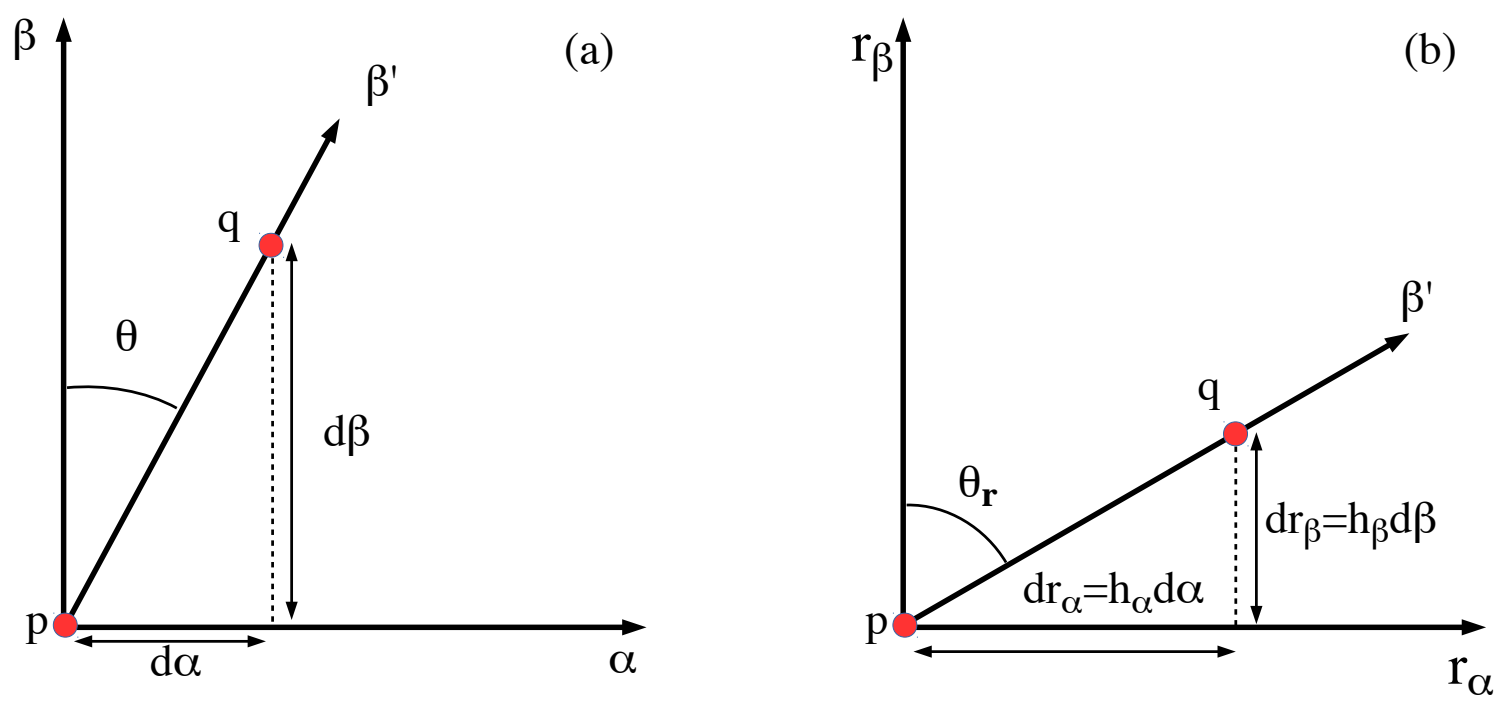

Figure 5. (a) Two field lines pass through the $(\alpha, \beta)$ plane as shown by the points $p$ and $q$ and define the $\beta^{\prime}$ axis. (b) In real space the $\beta^{\prime}$ direction makes a different angle to the $r_{\alpha}$ and $r_{\beta}$ directions. 
Equation (1) shows the relationship between scale factors and distances: two points separated along the $\beta$ axis by $d \beta$ with a scale factor $h_{\beta}$ will be separated by a real distance $d r_{\beta}=h_{\beta} d \beta$. We can turn this relation around and infer $h_{\beta}$ if we know the real separation of two points, $d r_{\beta}$. These ideas can be applied to the $\beta^{\prime}$ coordinate and used to derive a corresponding scale factor $h_{\beta^{\prime}}$ if we can calculate the separation of the points $p$ and $q$ - which is straightforward in terms of $\alpha, \beta, \gamma$ and their scale factors:

$$
\left(h_{\beta^{\prime}} d \beta^{\prime}\right)^{2}=\left(h_{\alpha} d \alpha\right)^{2}+\left(h_{\beta} d \beta\right)^{2}
$$

It is particularly important to determine how $h_{\beta^{\prime}}$ varies along the background field line (i.e., with $\gamma$ ). Consider evaluating (16) at an arbitrary value of $\gamma$ and also in a reference plane $\gamma=\gamma_{o}$ on the field line centered between $p$ and $q$ to give

$$
\begin{aligned}
\left(h_{\beta^{\prime}}(\gamma) d \beta^{\prime}\right)^{2} & =\left(h_{\alpha}(\gamma) d \alpha\right)^{2}+\left(h_{\beta}(\gamma) d \beta\right)^{2} \\
\left(h_{\beta^{\prime}}\left(\gamma_{o}\right) d \beta^{\prime}\right)^{2} & =\left(h_{\alpha}\left(\gamma_{o}\right) d \alpha\right)^{2}+\left(h_{\beta}\left(\gamma_{o}\right) d \beta\right)^{2}
\end{aligned}
$$

Here we use the shorthand where, for example, $h_{\beta^{\prime}}(\gamma)$ means the variation of $h_{\beta^{\prime}}$ with $\gamma$ on the field line considered. Dividing both of the above equations by $(d \alpha)^{2}+(d \beta)^{2}$, noting

$$
\sin ^{2} \theta=\frac{(d \alpha)^{2}}{(d \alpha)^{2}+(d \beta)^{2}}, \quad \cos ^{2} \theta=\frac{(d \beta)^{2}}{(d \alpha)^{2}+(d \beta)^{2}}
$$

and taking the ratio of the resulting two equations gives

$$
h_{\beta^{\prime}}(\gamma)=h_{\beta^{\prime}}\left(\gamma_{o}\right) \sqrt{\frac{h_{\alpha}^{2}(\gamma) \sin ^{2} \theta+h_{\beta}^{2}(\gamma) \cos ^{2} \theta}{h_{\alpha}^{2}\left(\gamma_{o}\right) \sin ^{2} \theta+h_{\beta}^{2}\left(\gamma_{o}\right) \cos ^{2} \theta}} .
$$

Finding the $h_{\alpha^{\prime}}$ scale factor needs a little care. Simply defining an $\alpha^{\prime}$ direction orthogonal to $\beta^{\prime}$ in the $\gamma=\gamma_{o}$ plane means these directions will not, in general, remain orthogonal in other surfaces of constant $\gamma$. A convenient way to infer a local scale factor for the transverse direction perpendicular to the $\beta^{\prime}$ direction in real space is by noting that in an orthogonal field aligned coordinate system the solenoidal constraint requires (Wright (1992))

$$
B h_{\alpha} h_{\beta}=B h_{\alpha^{\prime}} h_{\beta^{\prime}}=f(\alpha, \beta) .
$$

Since $f$ is constant on a field line we may use this equation to express the variation of $h_{\alpha^{\prime}}$ along a field line with the aid of (20),

$$
h_{\alpha^{\prime}}(\gamma)=\frac{h_{\alpha}(\gamma) h_{\beta}(\gamma)}{h_{\beta^{\prime}}\left(\gamma_{o}\right)} \sqrt{\frac{h_{\alpha}^{2}\left(\gamma_{o}\right) \sin ^{2} \theta+h_{\beta}^{2}\left(\gamma_{o}\right) \cos ^{2} \theta}{h_{\alpha}^{2}(\gamma) \sin ^{2} \theta+h_{\beta}^{2}(\gamma) \cos ^{2} \theta}}
$$

We can now write down the Alfvén wave equation for an Alfvén wave polarized along the $\beta^{\prime}$ axis - it is simply equation (3) with $\alpha$ and $\beta$ replaced by $\alpha^{\prime}$ and $\beta^{\prime}$ ( $\gamma$ and $h_{\gamma}$ are unchanged),

$$
\frac{\partial}{\partial \gamma}\left(\frac{1}{h_{\gamma}} \frac{\partial U_{\beta^{\prime}}}{\partial \gamma}\right)+\frac{1}{h_{\gamma}} \frac{\partial}{\partial \gamma}\left(\ln \left(\frac{h_{\beta^{\prime}}}{h_{\alpha^{\prime}}}\right)\right) \frac{\partial U_{\beta^{\prime}}}{\partial \gamma}+\frac{\omega_{A}^{2}}{V^{2}} h_{\gamma} U_{\beta^{\prime}}=0
$$

Equation (23) is a generalisation of the Alfvén wave equation for any polarisation angle $\theta$ : when $\theta=0$ or $\pi / 2$ it recovers the toroidal and poloidal wave equations given in (3) and (2), however, it also describes any intermediate polarisation. It is evident that the Alfvén frequency will vary smoothly with the polarization angle giving $\omega_{A}(\theta)$ on a particular field line. Equations (20), (22) and (23) are central to understanding the simulation results.

At first sight it may seem unlikely that the solution to equation (23) will manifest itself since $U_{\beta^{\prime}}$ must also be a solution of the governing equations (7)-(11) and part of the fast mode too. This is true in general. However, Wright (1992) has shown that when the length scale in $\alpha^{\prime}$ is suitably small (as it will be across a resonance) the perturbations are ordered in the following fashion: $U_{\beta^{\prime}} \sim B_{\beta^{\prime}} \gg U_{\alpha^{\prime}} \sim B_{\alpha^{\prime}} \gg B_{\gamma}$. Here $U_{\beta^{\prime}}$ is the solution of equation (23) and $B_{\beta^{\prime}}$ the associated magnetic field. Thus the dominant perturbations are indeed a resonant Alfvén wave. At leading order $\boldsymbol{\nabla} \cdot \mathbf{B}=0$ is satisfied by balancing the contributions from the two transverse field components - something we confirmed in our simulation results. The magnetic field compression $\left(B_{\gamma}\right)$ only enters at a higher order and is zero at 
the leading order of $U_{\beta^{\prime}}$ and $B_{\beta^{\prime}}$. This is in accord with Singer et al. (1981) who noted that the Alfvén wave equation decouples if $B_{\gamma} \approx 0$.

\section{2. $\omega_{A}$ for Arbitrary Polarisation}

Note that $\omega_{A}$ does not depend upon $\gamma$ : it is a property of the field line and the polarisation angle, so depends only upon the field lines labels $\left(\alpha\right.$ and $\beta$ ) and $\theta$. Figure 6(a) shows the variation of $\omega_{A}$ with $\theta$ for five different field lines. These curves can be used to interpret the simulation results in the $(\alpha, \beta)$ plane, which Figure 3 established are the natural coordinates for studying the resonance.
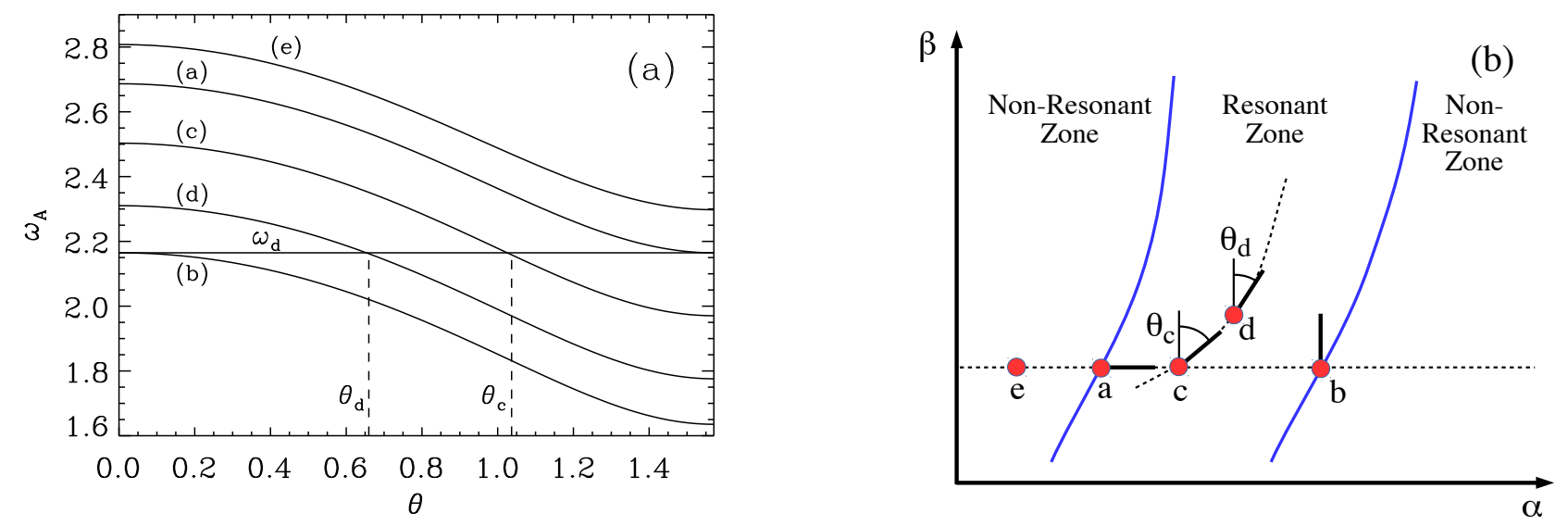

Figure 6. (a) The variation of $\omega_{A}$ with polarisation angle $\theta$. The horizontal line represents the value of the driving frequency $\left(\omega_{d}\right)$ which can match $\omega_{A}$ on some field lines if $\theta$ has an appropriate value (e.g., $\theta_{c}$ and $\left.\theta_{d}\right)$. (b) The existence of resonant and non-resonant zones, and the use of the polarisation angle to map out the resonant contour.

Figure $6(\mathrm{~b})$ shows the $(\alpha, \beta)$ positions of several fields lines labelled (a) to (e) and indicated by red dots. A shooting code was used to calculate the fundamental frequency $\left(\omega_{A}\right)$ for the indicated field lines as a function of $\theta$, and the results displayed in Figure 6(a). If the system is now driven at frequency $\omega_{d}$, which field lines are resonant, and at what polarisation angles $(\theta)$ ?

The horizontal line in the Figure 6 (a) denotes the driving frequency $\omega_{d}$. The intersection of this with a chosen $\omega_{A}(\theta)$ curve identifies the value of $\theta$ for which the Alfvén frequency matches the driving frequency on a particular field line. For the field line labelled (a), a resonance occurs when $\theta=\theta_{a}=\pi / 2$, and this direction is indicated by the short black line emerging from the red dot labelled (a) in Figure 6(b). Similarly, the intersection of the $\omega_{A}(\theta)$ curve for the field line labelled (b) with the $\omega_{d}$ line indicates the resonant polarisation is $\theta_{b}=0$, and is again indicated by the short line emerging from the red dot labelled (b) in Figure 6(b).

The horizontal dashed line in Figure 6(b) can be used to study how $\theta$ varies with $\alpha$ (at constant $\beta$ ) if we have the Alfvén frequency matching $\omega_{d}$. We have already established that this angle will change from 0 to $\pi / 2$ on moving from field line (b) to field line (a). For a suitably smoothly varying medium we find that the resonant angle will vary smoothly with intermediate values of $\alpha$. The exact value of $\theta$ is found from inverting the relation $\omega_{A}(\alpha, \beta, \theta)=\omega_{d}$ (depicted in Figure 6(a)) to give $\theta$ in terms of $\alpha, \beta$ and $\omega_{d}$. We shall see that it will be useful to define $\tan \theta \operatorname{through}$ this process,

$$
\tan \theta=F\left(\alpha, \beta, \omega_{d}\right) .
$$

For a suitable medium a monotonic variation of $\theta$ is found between field lines (a) and (b) (this is not essential, and only assumed for illustrative purposes), and the field line labelled (c) has an intermediate polarization $\left(\theta_{c}\right)$. Outside of the section between points (a) and (b) no solution for $\theta$ exists. For example, examining Figure $6(\mathrm{a})$ shows that field line (e) can never satisfy the resonance condition. If the horizontal dotted line in Figure 6(b) is shifted slightly to a new value of $\beta$ and the analysis repeated we can again identify the bounds of the region where the resonant condition can be satisfied. Extrapolating to all $\beta$ we can map out the bounds (shown in blue) to the Resonant Zone, outside of which the Non-Resonant Zone is found. We refer to Figure 6(b) as a "Resonance Map." 
How do these ideas explain the resonant features found in the simulation results shown in Figure 3? Suppose field line (c) on the Resonance Map is resonant with polarisation $\theta_{c}$. We can follow a path (shown as the curved dotted line in Figure 6(b)) at the polarisation angle $\theta_{c}$ which takes us to field line $(\mathrm{d})$. Once at (d) the resonant orientation changes slightly to $\theta_{d}$ and allows the dotted line to be continued further on in a similar fashion unless it reaches one of the boundaries of the Resonant Zone. By combining equations (14) and (24) we find an ordinary differential equation for the dotted line

$$
\frac{d \alpha}{d \beta}=F\left(\alpha, \beta, \omega_{d}\right)
$$

which may be integrated and the solution expressed in the form

$$
G\left(\alpha, \beta, \omega_{d}\right)=\text { constant. }
$$

Note the integration constant in (26) means there are a family of possible solutions: indeed field line (c) in Figure 6 could have been chosen anywhere between field lines (a) and (b) and a new path followed, confirming that a family of possible solutions exist. Moreover, we note that the scale factors in equations (20) and (22) are unchanged by replacing $\theta$ by $-\theta$ so the resonance condition will also be satisfied by introducing a sign change to equation (25) indicating that a second family of resonant curves also exist. This is somewhat unexpected as Alfvén resonances in 1D and 2D have a unique resonant solution, so the possibility an infinite family of permissible solutions in 3D represents a fundamental difference.

\subsection{Selection of Resonant Solutions}

The analysis of the previous section indicates that a family of resonant solutions can exist. However, the simulation results in Figure 3 indicate that a particular solution was preferred. In this subsection, we check whether the resonant ridge (from simulations) matches one of the solutions to equation (25) and identify the mechanisms that favour it.

\subsubsection{Boundary Conditions}

Figure 7(a) reproduces the energy density plot seen in Figure 3 but includes the bounds of the Resonant Zone (shown as the red lines) described in the previous subsection. Evidently the accumulation of energy is confined to the Resonant Zone. Figure 7(b) is the Resonance Map for this model and shows the bounds of the Resonant Zone (black dashed lines) as well as a selection of solutions to equation (25) as solid black lines. The path of the resonant ridge in Figure 7(a) is plotted in the Resonance Map (Figure 7(b)) as the solid green line and is indeed seen to be one of the family of possible resonant solutions. This means that everywhere along the green line the polarisation angle is just that required to make the corresponding Alfvén frequency match the driving frequency. Thus we have conclusive proof that this is an Alfvén resonance in a 3D medium.

Note how in Figure 7 the resonant solution avoids leaving the resonant zone by switching to the alternative solution (with the sign of $\theta$ changed) when it encounters the driven boundary at $(\alpha, \beta)=(1,0.15)$ or the boundary to the Resonant Zone $(0.9,0.1)$ and $(0.93,0.02)$. In this fashion the resonant solution avoids regions outside of the Resonant Zone, where no solution exists. We are not aware of this type of behavior having been identified in other studies. The resonant ridge from Figure 3(a) (shown as the green line in Figure 7(b)) clearly corresponds to one of the resonant paths from equation (25).

Why is this particular path selected? A clue comes from identifying the intersection of the green line with the inner boundary at $\alpha=0.5$ where we impose $U_{\alpha}=B_{\alpha}=0$ but $U_{\beta}$ and $B_{\beta} \neq 0$. Hence we are forcing the Alfvén wave velocity and magnetic field to be strictly in the $\beta$ direction. We know from Figure 4 that the Alfvén fields are aligned with the resonant contour, so the solution selected must have these fields polarised in the $\beta$ direction on the $\alpha=0.5$ boundary, which corresponds to $\theta=0$. The right hand boundary of the Resonant Zone corresponds to the solution with $\theta=0$ (as is the case for the sketch in Figure 6). Hence the solution passing through the intersection of this boundary with $\alpha=0.5$ is selected. Indeed this is exactly the point from which the observed solution (green line) originates.

We test the above hypothesis by modifying the boundary conditions and observing which solution our simulation provides. In particular we introduce a step in the dissipation coefficient at $\alpha=0.7$ shown as the dashed blue line in Figure 7. For $\alpha<0.7 \nu=1.0$ and effectively prevents any disturbance entering this region. The step in dissipation will allow $U_{\beta}$ and $B_{\beta}$ to be large immediately to the right of the dashed blue line (where $\nu$ is small), whilst $U_{\alpha}$ and $B_{\alpha}$ will be substantially smaller. This suggests the polarization on $\alpha=0.7$ will be $\theta=0$, so the resonant ridge should pass the point of intersection of the right hand boundary of the resonant zone with $\alpha=0.7$. A full numerical solution similar to that in Figure 7(a) was computed and the path of the ridge determined. The result is shown as the solid blue line, and indeed goes through the expected point. 

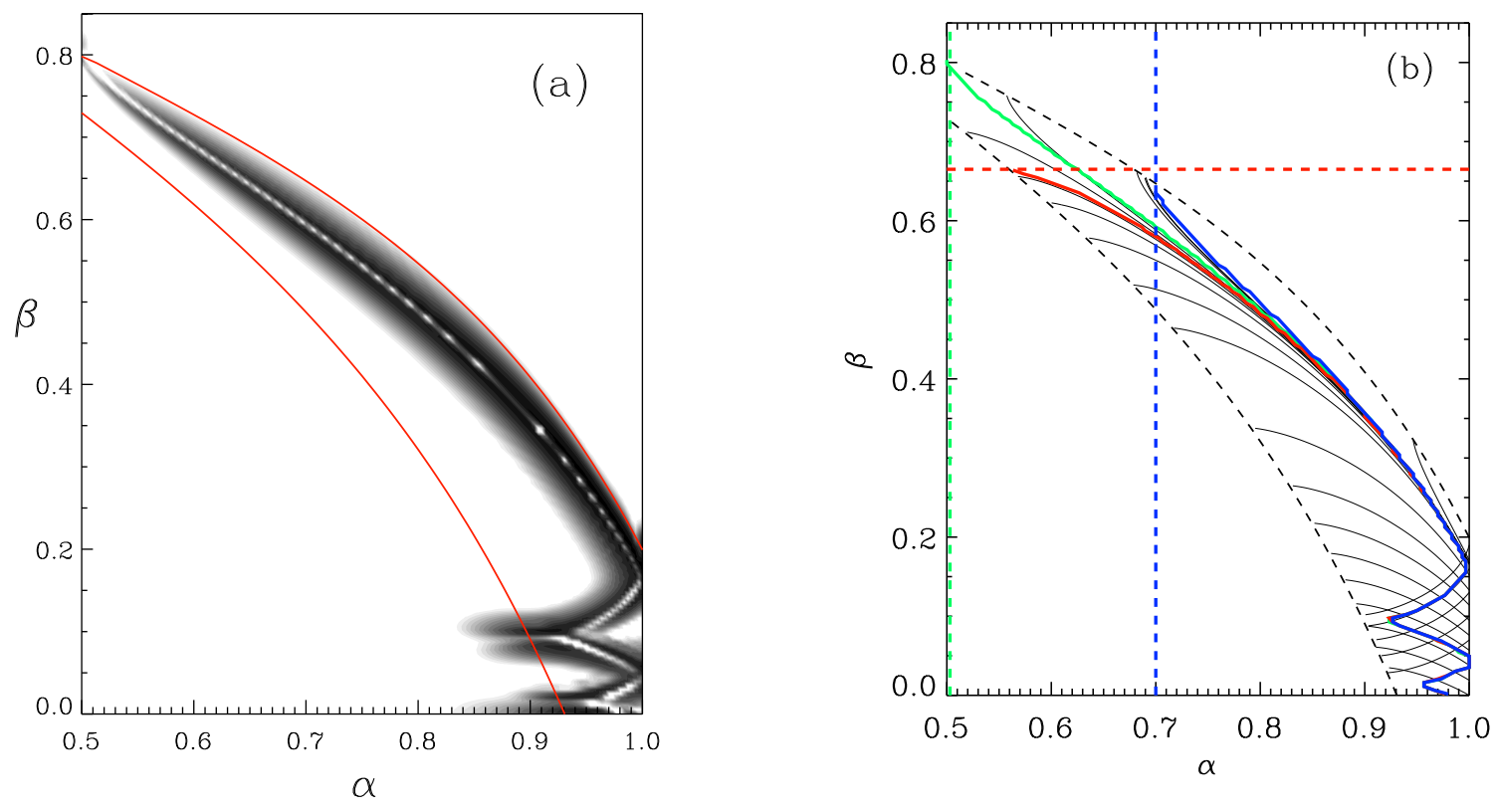

Figure 7. (a) Total energy density in the equatorial $\gamma=0$ plane with Resonant Zone bounds (red lines) included. (b) The Resonance Map in which the resonant ridge from panel (a) is shown in green, with other ridges (red and blue) shown for different boundary conditions. The bounds of the Resonant Zone are shown as the black dashed lines. The solid black lines are selected curves found as solutions to equation (25).

As a further test we removed the dissipative region in $\alpha<0.7$ but added one with a step centered on $\beta=0.67$ shown by the red dashed line. The large dissipation above the red dashed line effectively means that just below the line $U_{\alpha}$ and $B_{\alpha}$ may be large, whilst $U_{\beta}$ and $B_{\beta}$ will be much smaller. Hence $\theta=\pi / 2$, so the solution should go through the intersection of the left hand boundary of the Resonant Zone (corresponding to $\theta=0$ ) with the red dashed line. A similar numerical solution to that in Figure 7(a) was found and the path of the ridge determined. The result is shown in Figure 7(b) as the solid red line, and goes through the expected point. It is also evident that the different solutions converge rapidly and soon become indistinguishable at larger $\alpha$ where the energy density is largest.

\subsubsection{Locally $2 D$ regions}

The above subsection shows how boundary conditions play an important role in determining where the resonance forms. However, what happens if the Resonant Zone bounds do not intersect any simulation boundaries or equivalent features? To investigate this situation we introduce periodic boundary conditions so that the mechanism discussed in the previous subsection will not operate. The first set of results we present in this section uses $0.5<\alpha<1.0$, $0<\beta<0.4,0<\gamma<0.375, R_{g}=0.75, \nu=0.03, \omega_{d}=2.89741$ and a grid of $200 \times 150 \times 20$. Dissipative buffer zones are not needed for these results which are periodic in $\beta$. The boundary at $\alpha=1$ is again driven by prescribing the value of $B_{\gamma}$ which has a propagating nature in the $+\beta$ direction with a wavelength of 0.4 , and has a similar profile in $\gamma$ to the previous simulations.

In Figure 8(a) the diffuse shading extending from the driven boundary along the whole extent of $\beta$ is associated with the evanescent fast mode excited at the driven $(\alpha=1)$ boundary. The narrower criss-cross features centered around $(0.85,0.3)$ are associated with the excitation of resonant Alfvén waves. These have their largest amplitude at $(0.91,0.3)$ which, intriguingly, is where the toroidal mode (the right hand side of the Resonant Zone) has a turning point with respect to $\beta$. This leads us to speculate that, even though our medium is fully $3 \mathrm{D}$ with no extended 2D sections anywhere, the locally $2 \mathrm{D}$ nature of the medium at the toroidal mode turning point does seem to favour strong resonant coupling. There will be a similar locally $2 \mathrm{D}$ region at $(0.7,0.1)$, however coupling is weak here due to the evanescent nature of the fast mode.

The Resonance Map in Figure 8(b) indicates that the ridges in (a) do indeed align with the expected resonant contours. Of course, a resonant contour close to $(0.91,0.3)$ cannot progress very far in the $\beta$ direction before hitting the boundary of the Resonant Zone. When this happens the process identified in the previous subsection operates, and the resonant solution switches to another of the resonant contours that lies within the Resonant Zone. This accounts for the criss-crossing features emerging from $(0.91,0.3)$. 

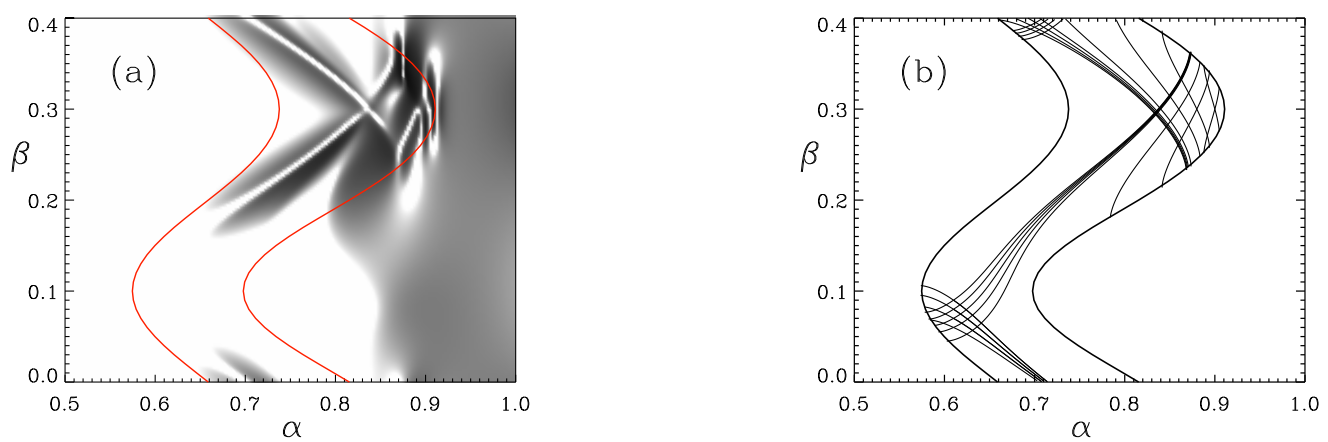

Figure 8. (a) Total energy density in the $\gamma=0$ plane with the Resonant Zone boundaries shown in red. (b) The Resonance Map for panel (a) showing Resonant Zone boundaries along with selected permissible resonant solutions.

These results suggest that locally $2 \mathrm{D}$ regions may play a significant role in interpreting our results. To explore the importance of both extended and local 2D regions in determining the formation of Alfvén resonances we present two final numerical experiments. These both have an extended section where the medium is properly $2 \mathrm{D}$ in addition to a turning point (locally 2D). Both sets of results have $0.5<\alpha<1.0,-0.4<\beta<0.4,0<\gamma<0.375, R_{g}=0.75$, $\nu=0.02, \omega_{d}=2.89741$ and a grid of $280 \times 180 \times 25$.

Figure 9 (a) shows the boundaries of the resonant zone as red lines. The background equilibrium is chosen to have a $2 \mathrm{D}$ variation (invariant in $\beta$ ) for $\beta<-0.2$ and $\beta>0.2$, and be periodic in $\beta$. Wright and Thompson (1994) have shown that in these regions there is a unique resonance with polarization $\theta=0$, which corresponds to the right hand side of the Resonant Zone here (around $\alpha=0.91$ ). It is natural that this location should see strong Alfvén wave coupling and the numerical solution (Figure 9(a)) confirms this.

The Resonance Map in Figure 9(b) shows the corresponding Resonant Zone boundaries along with selected solutions to equation (25). The simulation results in Figure 9(a) also show additional resonant ridges coming from $(\alpha, \beta) \approx$ $(0.7,0.0)$ which appear to be following a different resonant solution. This solution passes through the intersection of the $\beta=0$ line and the right hand $(\theta=0)$ Resonant Zone boundary. On the $\beta=0$ line we have $\partial V / \partial \beta=0$, so the medium is locally $2 \mathrm{D}$ suggesting we should establish a solution with $\theta=0$ which explains the prominence of the solution passing through $(0.7,0.0)$. Note that this solution is much smaller than the resonance at $\alpha=0.91$, presumably because the fast mode has to tunnel further to get to $\alpha=0.7$. (The amplitude of the fields at $(\alpha, \beta) \approx(0.91,0.3)$ exceeded those near $(0.7,0)$ by a factor of around 12 .)
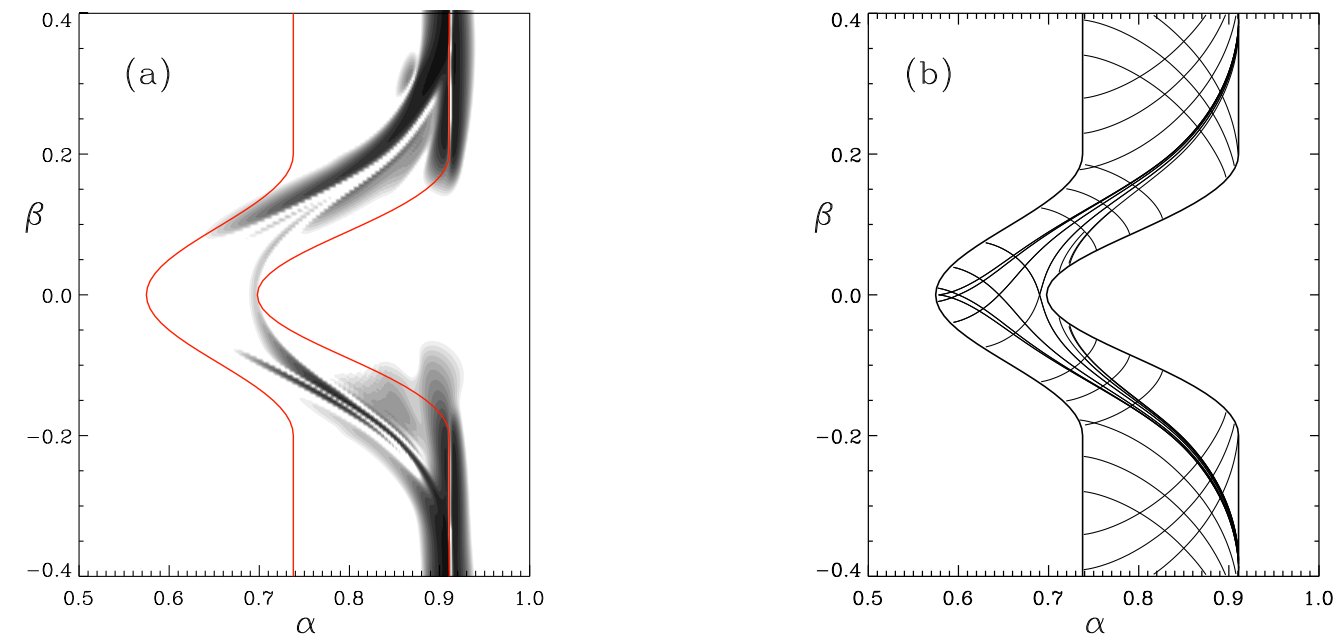

Figure 9. (a) Total energy density in the $\gamma=0$ plane with the Resonant Zone boundaries shown in red. (b) The Resonance Map for panel (a) showing Resonant Zone boundaries along with selected permissible resonant solutions.

The Alfvén speed variation was adjusted so that the extended $2 \mathrm{D}$ sections are now at $\alpha=0.7$, while the locally $2 \mathrm{D}$ region is at $(\alpha, \beta)=(0.91,0.0)$, as displayed in Figure 10. As expected, a resonance is found at $\alpha=0.7$ in the 2D 

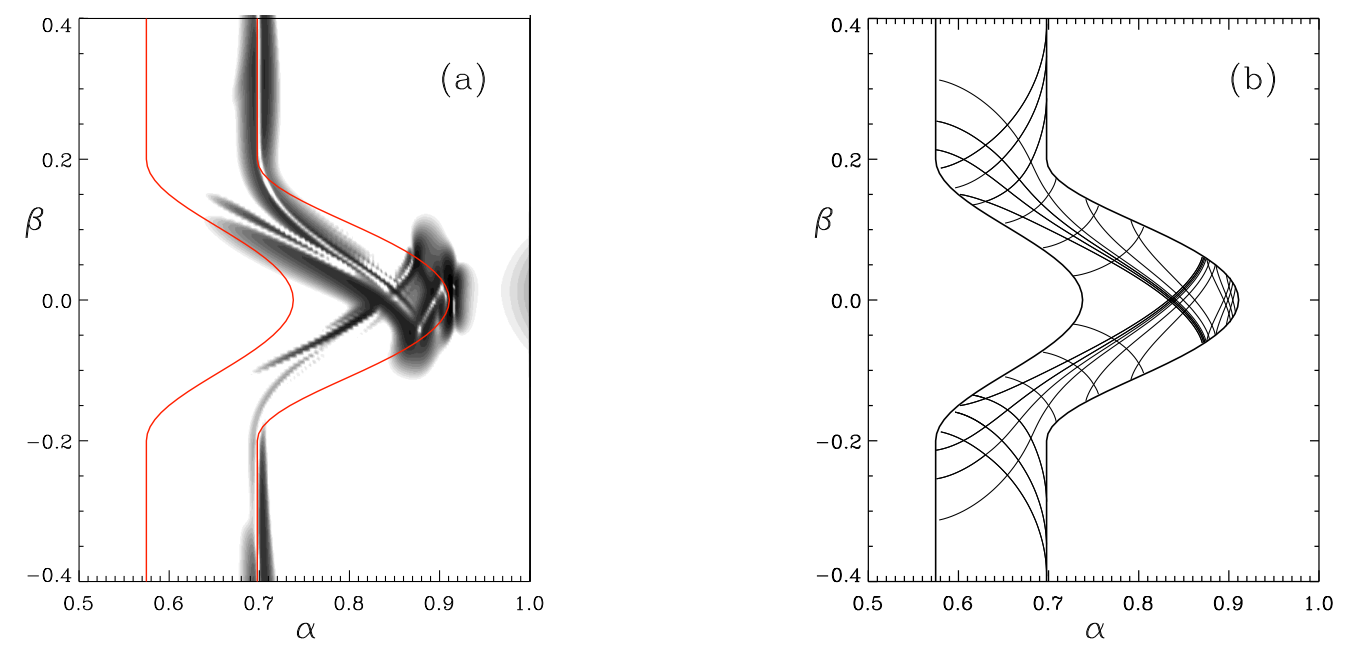

Figure 10. (a) Total energy density in the $\gamma=0$ plane with the Resonant Zone boundaries shown in red. (b) The Resonance Map for panel (a) showing Resonant Zone boundaries along with selected permissible resonant solutions.

sections. Also the locally $2 \mathrm{D}$ section at $(\alpha, \beta)=(0.91,0.0)$ selects a resonant solution (with $\theta=0$ ), and this exhibits a criss-cross pattern as the solution switches when it encounters the boundary of the Resonant Zone. Note that the amplitude of the solution at $(0.91,0.0)$, despite only being locally $2 \mathrm{D}$, dominates that at $\alpha=0.7$ by a factor of about 5 as the fast mode decays in amplitude from the $\alpha=1.0$ boundary. Clearly having an extended 2D region is not the most important factor in determining where strong resonant coupling will occur.

It should be stressed that we had no particular motivation for introducing $2 \mathrm{D}$ or locally $2 \mathrm{D}$ (turning points) to our equilibrium: they are simply unavoidable in a periodic domain. This will apply equally to, for example, a planetary magnetosphere which will be periodic in the longitudinal coordinate. Indeed, it may be appreciated intuitively that a simple magnetosphere with day-night asymmetry would have turning points in the Resonant Zone in the noon and midnight meridians. In a solar context, a simple arcade that has nonuniformity in the magnetic field or plasma density along its length will find it hard to avoid having maxima or minima in the Resonant Zone boundaries, and hence turning points. Our results indicate that such locations can play an important role in determining the sites of strong resonant coupling.

\section{DISCUSSION AND CONCLUSIONS}

To interpret resonances in 3D we have found it helpful to construct a Resonance Map depicting the Resonant Zone and the Non-Resonant Zone. It is not possible for the Alfvén frequency $\left(\omega_{A}\right)$ of field lines in the Non-Resonant Zone to match the driving frequency $\left(\omega_{d}\right)$. For field lines in the Resonant Zone it is possible to have $\omega_{A}=\omega_{d}$, however this will only be possible for a particular polarisation of the Alfvén wave which may vary anywhere between the extremes of toroidal and poloidal.

For our simple 3D medium the boundaries of the Resonant Zone boundaries correspond to the poloidal and toroidal polarisations. For a more general medium this may not be the case, nor would it be true if the transverse coordinates were chosen differently. Nevertheless our procedure of identifying the Resonant Zone can still be followed: a figure similar to that in Figure 6 would need to be constructed over the range $0<\theta<\pi$ and the turning points that have a frequency equal to $\omega_{d}$ would identify the field lines and the values of $\theta$ that correspond to the polarisation on the boundaries of the Resonant Zone. The dominant Alfvén fields on a resonance have been shown to lie in the resonant surface (Figure 4), which is also the case in 1D and 2D resonances.

Alfvén resonances in $3 \mathrm{D}$ are fundamentally different to those in $1 \mathrm{D}$ and $2 \mathrm{D}$ in that there is not a unique resonant solution, but a family of possible resonant surfaces that lie within the Resonant Zone. It may be that a general forcing of the system at a particular frequency $\left(\omega_{d}\right)$ will excite all these solutions to some extent, but some are favored and dominate the overall response. In particular we have identified that boundary conditions can play an important role and favour the resonant solution that is consistent with them. Another process that allows a particular solution to dominate is if the medium contains $2 \mathrm{D}$ portions (even if just locally in the form of turning points) where existing 2D theories identify a single resonant solution.

A unique feature of 3D resonances is the possibility that a favored resonant solution may encounter the boundaries 
of the Resonant Zone. Of course, it is not possible for such a solution to cross the boundary and exist in the NonResonant Zone. Our numerical solutions show how the resonant fields switch to following an alternative solution at the boundary such that the resonance can continue within the Resonant Zone. The Alfvén resonance is not cut off abruptly at the boundary and appears to have an evanescent-like feature tunneling into the Non-Resonant Zone.

Both authors were funded in part by STFC (through Consolidated Grant ST/N000609/1) and The Leverhulme Trust (through Research Grant RPG-2016-071).

\section{REFERENCES}

Allan, W., White, S. P., \& Poulter, E. M. 1986, Planet. Space Sci., 34, 371

Andries, J., Goossens, M., Hollweg, J. V., et al. 2005, Astron. Astrophys., 430, 1109

Arregui, I., \& Ballester, J. L. 2011, Space Sci. Rev., 158, 169

Chen., L., \& Hasegawa, A. 1974, J. Geophys. Res., 79, 1033

Claudepierre, S. G., Hudson, M. K., Lotko, W., et al. 2010, J. Geophys. Res., 115, A11202

Degeling, A. W., Rankin, R., Kabin, K., Rae, I. J., \& Fenrich, F. R. 2010, J. Geophys. Res., 115, 10212

Elsden, T. 2016, PhD. Thesis, University of St Andrews

Elsden, T., Wright, A. N. 2016, J. Geophys. Res., in preparation

Goossens, M., Ruderman, M, \& Hollweg, J. V. 1995, Sol. Phys., 157,75
Hansen, P. J., \& C. K. Goertz 1992, Phys. Fluids B, 4, 2713

Mann, I. R., Wright, A. N., \& Cally, P. S. 1995, J. Geophys. Res., 100, 19441

Russell, A. J. B., \& Wright, A. N. 2010, Astron. Astrophys., 511, A17

Singer, H. J., Southwood, D. J., Walker, R., J., \& Kivelson, M. G. 1981, J. Geophys. Res., 86, 4589

Soler, R., \& Terradas, J., 2015, ApJ, 803, 43

Southwood, D. J. 1974, Planet. Space Sci, 22, 483

Terradas J., Soler, R., Luna, M., Oliver, R., Ballester J. L., \& Wright, A. N. 2016, ApJ, 820, 2

Wright, A. N. 1990, J. Plasma Phys., 43, 83

Wright, A. N. 1992, J. Geophys. Res., 97, 6439

Wright, A. N., \& Thompson. M. J. 1994, Phys. Plasmas, 1, 691 